\title{
THE THEORY OF NUMBERS.-I.
}

Sur la théorie des nombres.* M. Straltums. (Annales de la Fraculté des Sciences de Toulouse, vol. 4.)

M. STIELTJEs undertook a few years ago to write an extensive treatise on the theory of numbers. His weak health, and finally untimely death, prevented the accomplishment of his task. The following letter which I received from him in answer to a request to send me a separate copy of his paper will put the circumstances clearly before the reader:

“Toulouse, May 29, 1894.

"I am exceedingly sorry not to be able to send you a copy of my paper. When I undertook this work it was my intention to carry it much farther, and to publish several volumes. I had the first volume nearly ready, and it was agreed that M. Gauthier-Villars should publish it separately. So I did not receive any separate reprints of the part already published, probably because M. Gauthier-Villars intended to send them to me when the first volume should be finished. But in proportion as I advanced, it became more and more evident that I would not be able to gather together in Toulouse all the materials for my work. I had made up a list of about thirty papers which it would be impossible for me to get, and which were absolutely necessary in order to finish the first volume. I lost heart, my health gave way too, so that I was obliged to spend two winters in Algiers,-and I could not work. So that it is perfectly certain that I shall not resume the task in the future; the idea of doing it is very far from me now. And then my work was nothing else but a bibliographical review, and I only summed up what had been done by others, in particular by H. J. Stephen Smith.

"Going back to the sources, accordingly, you will advantageously supply the absence of my work, which had no other object but to be useful to beginners who know little or nothing of the theory of numbers. To prove you my goodwill, I hope you will permit me to send you a paper on continued fractions, which I have just brought painfully to a close. But it will not appear before the end of the year. $\dagger$

* Also now published separately. See list of Nero Publications in this number of the Bulletrin, p. 239 .

$\uparrow$ This promise was faithfully fulfilled. The paper appeared in the eighth volume of the Annales de Toulouse. 
To avoid your being disappointed at that time, I shall state at once that it deals with algebra and analysis, and not with the theory of numbers.

" Pray accept, etc.

T. Stieltues."

The oldest treatise on the theory of numbers that has come down to us is the one contained in the Serenth, Eighth, and Ninth Books of Euclid's Elements.* The first principles of our science will be found there exposed "with the usual rigor and terseness of the ancients." The subjects treated by Euclid, such as the independence of a product of the order of its factors, $\uparrow$ the rule for finding the greatest common measure, numbers prime to one another, least common multiples and greatest common divisors, the decomposition of a number into prime factors and its uniqueness, and finally the proposition that our stock of prime numbers can never be exhausted, might be properly designated under the name of Euclidian arithmetic.

We shall say accordingly that the beginning of M. Stieltjes' paper is devoted to Euclidian arithmetic, greatly generalized, however. He does not insist on the definition of number $\ddagger$ nor on the laws which are at the base of the operations we perform on numbers, $\S$ but after a simple mention of these topics, passes immediately to the exposition of the chief properties of the least common multiple and the greatest common divisor of numbers. Euclid gives a rule for finding the greatest common divisor of two numbers, $\|$ and reduces to it the problem of finding the least common multiple of two numbers. $\uparrow$ Poinsot** was the first, I think, to whom it occurred that the course could be reversed. I shall present his process in a somewhat more symmetrical form. Suppose

* I shall always refer to Dr. Heiberg's edition, where the Books mentioned will be found on pages 184-437 of the second volume.

+ The associative law is not mentioned-a deficiency happily supplied by the Fifth Book, which is thus stamped at once as coming from another hand (Eudoxus). Gauss was the next to consider the associative law in his theory of composition of forms-and he quotes the Fifth Book of the Elements (Disqu. Ar., art. 239).

$\ddagger$ On this subject see Helmholtz's and Kronecker's papers in the Philosophische Aufsätze an Zeller (Leipzig, 1887), and Professor Dedekind's pamphlet, Was sind und was sollen die Zahlen (Braunschweig, 1888).

§ See Grassmann's Arithmetik (Stettin-I think most copies have Berlin on the title-page-1860) and Clifford's Common-Sense of the Exact Sciences.

IEuclidis Elementa, vir., 1, 2.

T Eucl. Elem. vir., 34.

** Journal de Liouville, 1845, p. 48. 
we have $a$ rows of $b$ points, placed so as to form at the same time $b$ columns of $a$ points:

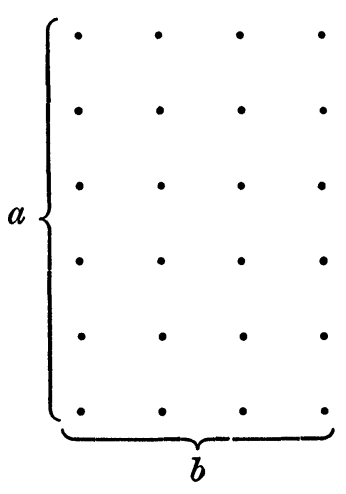

We can now cut out the slip of paper containing the points, deform it, and paste on the surface of an anchor ring, so that each column will be on a parallel and each row on a meridian. Let us now start from any point of the configuration-say the lower left-hand corner, using still our plane representation to facilitate the explanation - and then make a move forwards and to the right, then another move in the same direction, and so forth, keeping well in mind that the

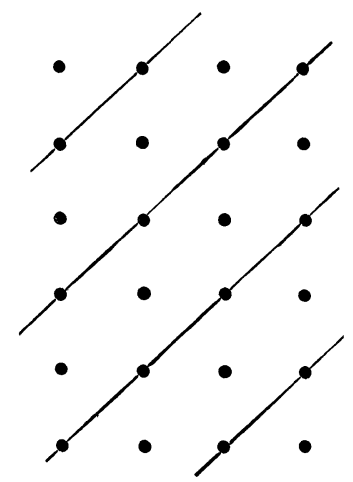

whole configuration is on an anchor ring. As the stock of points is a limited one,-there are $a b$ points in all,-we must finally return to some point where we have been before. I say it will be the initial point.

In fact, there are two elementary movements to be con- 
sidered-the one by which all parallels revolve so as to coincide each with its old position, while each meridian is replaced by the next one, and the other such that each meridian coincides with its old position, while each parallel is replaced by the next one. Now it is clear that if after several such movements the point $A^{\prime}$ comes to coincide with the point $A$, and the points $B$ and $B^{\prime}$ are the points to which we should move from $A$ and $A^{\prime}$ according to the rule described above, then when $A^{\prime}$ coincides with $A, B^{\prime}$ must coincide with $B$. Now if we perform the requisite movements in order to bring the point to which we returned in coincidence with our initial point, then, unless our proposition is true, the course described starting from the initial point does not coincide with the course obtained by following the track of the point which is now in coincidence with the initial point, which is absurd.

Let $h_{1}{ }^{\prime}, h_{2}{ }^{\prime}, \ldots h_{m}{ }^{\prime}$ be the points through which we passed in succession, so that one step more would bring us again to the initial point $h_{1}^{\prime}$. Then if $m=a b$ our supply of points is exhausted. If $m<a b$, let $h_{1}^{\prime \prime}$ be a point through which we did not pass. By taking $h_{1}^{1}$ " as initial point we obtain a cycle of points:

$$
h_{1}^{\prime \prime}, h_{2}^{\prime \prime}, \ldots h_{u}^{\prime \prime}
$$

I say $u$ must be equal to $m$, because if we bring $h_{1}^{\prime \prime}$ in coincidence with $h_{1}^{\prime}$ by a combination of movements described above, the two cycli

and

$$
h_{1}^{\prime}, h_{2}^{\prime}, \ldots h_{m}^{\prime}
$$

$$
h_{1}^{\prime \prime}, h_{2}{ }^{\prime \prime}, \ldots h_{u}{ }^{\prime \prime}
$$

will coincide, which gives

$$
u=m \text {. }
$$

The two cycli can have no common point, because otherwise there would be two divergent tracks from such a point, which is absurd on account of the perfect determinateness of the track of a point. If $2 m=a b$ our stock is exhausted; if $2 m<a b$ let $h_{1}{ }^{\prime \prime \prime}$ be a point not on the two tracks already described. The point $h_{1}^{\prime \prime \prime}$ will give us a new track,

$$
h_{1}{ }^{\prime \prime \prime}, h_{2}{ }^{\prime \prime \prime}, \ldots h_{m}{ }^{\prime \prime \prime} \text {, }
$$


and so forth, till the tracks described

$$
\begin{aligned}
& h_{1}{ }^{\prime}, h_{2}{ }^{\prime}, \ldots . . h_{m}{ }^{\prime} \\
& h_{1}{ }^{\prime \prime}, h_{2}{ }^{\prime \prime}, \ldots . h_{m}{ }^{\prime \prime} \\
& h_{1}{ }^{\prime \prime \prime}, h_{2}{ }^{\prime \prime \prime}, \ldots h_{m}{ }^{\prime \prime \prime} \\
& \text {. . . . . } \\
& \dot{h}_{i}^{(n)}, \dot{h_{2}^{(n)}} \cdot \ldots \dot{h_{m}(n)}
\end{aligned}
$$

will include all the points of the configuration, so that

$$
a b=n m .
$$

In its arithmetical form this process is a very primitive one (logically, not historically), but perfectly sufficient to establish the chief properties of Euclidian arithmetic.

The numbers $a$ and $b$ being two given integers, I divide the succession of numbers

$$
1,2,3, \ldots
$$

by $a$ and $b$ till I come to a number $m$ such that the two divisions will leave no remainders, which must happen sooner or later, because the number $a b$ is divisible both by $a$ and $b$. It may happen that $m=a b$ so that $a b$ is the least number divisible by $a$ and $b$. If not, and accordingly $m<a b$, I write under the succession of numbers

$$
1,2,3, \ldots m
$$

another succession of numbers,

$$
m+1, m+2, m+3, \ldots 2 m \text {. }
$$

If a number of the first succession is divisible by $a$ and $b$, so will be the corresponding number of the second succession by virtue of the law of distribution. Now $m$ being the only number of the first succession divisible by $a$ and $b, 2 m$ will be the only number of the second succession divisible by $a$ and $b$. Now $a b$ being divisible by $a$ and $b$, it may happen to be equal to one of the two numbers $m$ and $2 m$, but cannot be equal to any other number of the two successions. The first equality having been already excluded, $2 \mathrm{~m}$ is the only number of the two successions to which $a b$ may happen to be equal now. If not, and accordingly $a b>2 \mathrm{~m}$, I write down another succession of numbers,

$$
2 m+1,2 m+2, \ldots 3 m,
$$


under the two first, and by reasoning in the same way as before we come to the conclusion that $3 \mathrm{~m}$ is the only number of the three successions to which $a b$ may now happen to be equal. By continuing in the same way we shall finally exhaust all the numbers

$$
1,2,3 . \ldots a b
$$

say after having written down $n$ successions of numbers, so that

$$
m n=a b .
$$

The number $m$ will be the least common multiple of $a$ and $b$, and it remains to show that $n$ is the greatest common divisor of $a$ and $b$. In fact, $m$ being a multiple of $a$, it follows from the equality

$$
m=a \times \frac{b}{n}
$$

that $n$ is a divisor of $b$. In the same way it follows from

$$
m=b \times \frac{a}{n}
$$

that $n$ is a divisor of $a$. If $n$ is not the greatest common divisor of $a$ and $b$, let $h$ be the greatest. Then it follows from

that

$$
h>n
$$

$$
\frac{a b}{h}<m
$$

and as $\frac{a b}{h}$ is an integer and a multiple of $a$ and $b$, in virtue of the equalities

$$
\frac{a b}{h}=a \times \frac{b}{h}=b \times \frac{a}{h},
$$

it would be a multiple of $a$ and $b$ smaller than $m$, contrary to what has already been proved.

Every multiple $k$ of $a$ and $b$ is a multiple of $m$. In fact, I write the succession of numbers

$$
1,2,3, \ldots k
$$

in rows of $m$ numbers,

$$
\begin{aligned}
& 1, \quad 2, \quad 3, \ldots m \\
& m+1, m+2, m+3, \ldots 2 m \\
& 2 m+1,2 m+2,2 m+3, \ldots 3 m \text {, }
\end{aligned}
$$


till I reach the number $k$, which can find its place only in the last column, because the numbers of the other columns are not divisible by $a$ and $b$ at the same time. The number $k$ will be equal accordingly to a multiple of $m$ such as $l m$.

It follows that every divisor $v$ of $a$ and $b$ is a divisor of $n$. In fact, $\frac{a b}{v^{\prime}}$ being a multiple of $a$ and $b$ will be a multiple of their least common multiple $\frac{a b}{n}$, and according $n$ divisible by v.

The converse propositions that every multiple of $m$ is a multiple of $a$ and $b$ and every divisor of $n$ is a divisor of $a$ and $b$ are of a still more elementary kind.

Two numbers whose greatest common divisor is equal to unity are said to be prime to one another. Such are, for instance, the numbers 8 and 15 . The least common multiple of two numbers $a$ and $b$ prime to one another is equal to $\frac{a b}{1}=a b$.

The important proposition VII., 30 of Euclid can now be easily established in a somewhat generalized form.

If $a$ is prime to $b$ and divides $b c$, then $a$ divides $c$. In fact, $a$ and $b$ being prime to one another their least common multiple will be equal to $a b$. Now $b c$ being a multiple of $a$ and $b$ will be a multiple of their least common multiple $a b$, and accordingly $c$ is a multiple of $a$. These propositions are generally proved by means of Euclid's rule for finding the greatest common divisor of two numbers.*

Among those who have adopted a point of view similar to the one exposed here, I may quote Professor Bachmann (Zahlentlieorie, 1892) and M. Emile Borel (Introduction à l'étude de la théorie des nombres, 1895).

Euclid's rule for finding the greatest common divisor $\nmid$ is so well known that I need not insist upon it. Its importance can hardly be overestimated, and up to the present day it is the only convenient rule for finding the greatest common divisor of two numbers. The other rule-usually given in the text-books-reduces the problem to a harder one, the individual decomposition of each number into factors.

The least common multiple of $a$ and $b$ being equal to $m$, the least common multiple of $a c$ and $b c$ will be equal to $m c$. In fact, $m c$ is evidently a multiple of $a c$ and $b c$. If these two numbers admitted a multiple $t<m c$, then $t$ would be of the form $k c$ where $k<m$ and a multiple of $a$ and $b$, which is

* Grassmann's Arithmetik, p. 40.

t Eucl. Elem., vir. 1, 2. 
absurd. It follows that if $n$ is the greatest common divisor of $a$ and $b$ then $n c$ is the greatest common divisor of $a c$ and $b c$.

The definition of the least common multiple and the greatest common divisor can be easily extended so as to apply to the case of more than two numbers

$$
a_{1}, a_{2}, \ldots a_{n}
$$

M. Stieltjes uses the very convenient designation

$$
\left|a_{1}, a_{2}, \ldots a_{n}\right|
$$

for the least common multiple and

$$
\left(a_{1}, a_{2}, \ldots a_{n}\right)
$$

for the greatest common divisor.

These two arithmetical functions are independent of the order of the numbers

$$
a_{1}, a_{2}, \ldots a_{n},
$$

and it is also evident that if we repeat any one of our numbers several times, the two functions will not change.

Let us now consider the succession of numbers

$$
1,2, \ldots m \text {, }
$$

where the last number $m$ is the first which is divisible by all the numbers

$$
a_{1}, a_{2}, a_{3}, \ldots a_{n} \text {. }
$$

That we must finally reach such a number $m$ follows from the fact that the product $a_{1} a_{2} \ldots a_{n}$ is divisible by $a_{1}$, $a_{2}, \ldots a_{n}$, although there may be smaller numbers having the same property. It is now easily proved that every multiple $\mu$ of

$$
a_{1}, a_{2}, \ldots a_{n}
$$

is a multiple of $m$ by writing down the rows

$$
\begin{gathered}
1, \quad 3, \ldots m, \\
m+1, \quad m+2, \quad m+3, \ldots 2 m, \\
2 m+1,2 m+2,2 m+3, \ldots 3 m,
\end{gathered}
$$

till we reach the number $\mu$, which can find its place only in the last column, because the numbers of the other columns are not divisible by all the numbers $a_{1}, a_{2}, \ldots a_{n}$. The proof of the converse proposition that every multiple of $m$ is a multiple of

is immediate.

$$
a_{1}, a_{2}, \ldots a_{n}
$$


By examining all the numbers in succession from one up to the smallest of the numbers

$$
a_{1}, a_{2}, \ldots a_{n},
$$

we can easily pick out all the common divisors

of the numbers

$$
\delta_{1}, \delta_{2}, \ldots \delta_{h}
$$

Let us now put

$$
a_{1}, a_{2}, \ldots a_{n}
$$

As all the numbers

$$
\left|\delta_{1}, \delta_{2}, \ldots \delta_{h}\right|=d \text {. }
$$

are multiples of

$$
a_{1}, a_{2}, \ldots a_{n}
$$

$$
\delta_{1}, \delta_{2}, \ldots \delta_{h},
$$

they will be multiples of their least common multiple $d$, and accordingly $d$ will be equal to the greatest of the numbers

$$
\delta_{1}, \delta_{2}, \ldots \delta_{h}
$$

This establishes not only the existence of the greatest common divisor $d$ of the numbers

$$
a_{1}, a_{2}, \ldots a_{n},
$$

but also the fact that every divisor of these numbers is a divisor of $d$.

In order to find the least common multiple $m$ of several numbers

$$
a_{1}, a_{2}, \ldots a_{n},
$$

we may replace any two of them $a_{h}$ and $a_{k}$ by their least common multiple $\left|a_{h}, a_{k}\right|$; then the problem of finding the least common multiple of

$$
a_{1}, a_{2}, \ldots a_{n}
$$

will be reduced to the problem of finding the least common multiple of the new row of $n-1$ numbers. In fact, every multiple of $a_{h}$ and $a_{k}$ is a multiple of $\left|a_{h}, a_{k}\right|$, and conversely. The number of the numbers of the new row is now equal to $n-1$, and can be diminished by one by repeating the process, and so on until we come to the single number $m$ as the final result. The process is an unsymmetrical one, but as the result can be expressed in a symmetrical form, it is independent of the order in which the operation is performed.

A similar process can be given for the finding of the greatest common divisor of several numbers.

We shall now establish a lemma which will be useful to us in the future. 
If $b$ and $c$ are prime to one another, then the greatest common divisor of $a$ and $b c$ is equal to the product of the greatest common divisor of $a$ and $b$ by the greatest common divisor of $a$ and $c$.

$$
\begin{aligned}
& \text { Demonstration.- } \\
& \qquad \begin{aligned}
(a, b c) & =(a b, a c, b c)^{*}=(a b, c(a, b)) \dagger=(a c, b(a, c)) \ddagger \\
& =(a b, a c, c(a, b), b(a, c))=(b(a, c), c(a, b)) \\
& =(a, b) \times(a, c) . \S
\end{aligned}
\end{aligned}
$$

The proposition may be immediately extended to the case of any number of numbers prime each to each,

so that we shall have

$$
b, c, d, \ldots,
$$

$$
(a, b c d \ldots)=(a, b) \times(a, c) \times a, d) \times \ldots
$$

It happens very often in the theory of numbers, as well as in the theory of groups, that the least common multiple $m$ of $n$ numbers

has to be decomposed into $n$ factors

$$
a_{1}, a_{2}, \ldots a_{n}
$$

$$
m=b_{1} b_{2} \ldots b_{n}
$$

such that each $b$ divides the corresponding $a$, and the factors are prime each to each.

The problem may be solved in the following way: If no two $a$ 's have a common factor, then

and we can put

$$
m=a_{1} a_{2} \ldots a_{n},
$$

$$
b_{1}=a_{1}, \quad b_{2}=a_{2} \ldots b_{n}=a_{n}
$$

If two of the $a$ 's, say $a_{h}$ and $a_{k}$, have a common factor $d$, and that factor happens to be prime to $\frac{a_{h}}{d}$, then, as $\frac{a_{h}}{\bar{d}}$ is prime to $\frac{a_{k}}{d}$ and $d$, it will be prime to their product $\| a_{k}$, and accordingly $\frac{a_{h} a_{k}}{d}$ will be the least common multiple of $\frac{a_{h}}{d}$ and

\footnotetext{
* Because the greatest common divisor of $a b$ and $a c$ is $a$.

$\uparrow$ Because the greatest common divisor of $a c$ and $b c$ is $c$ times the greatest common divisor of $a$ and $b$.

$\ddagger$ For a similar reason.

$\$$ Because the product evidently divides both $b .(a, c)$ and $c .(a, b)$ and leaves two quotients, $\frac{b}{(a, b)}$ and $\frac{c}{(a, c)}$, prime to one another, on account of $b$ and $c$ being so.

\| Eucl. Elem. vIr. 24.
} 
$a_{k}$. So that if we replace the row of numbers

by

$$
a_{1}, a_{2}, \ldots a_{h-1}, a_{h}, a_{h+1}, \ldots a_{k-1}, a_{k}, a_{k+1}, \ldots a_{n}
$$

$$
a_{1}, a_{2}, \ldots a_{h-1}, \frac{a_{h}}{d}, a_{h+1}, \ldots a_{k-1}, a_{k}, a_{k+1}, \ldots a_{n},
$$

the least common multiple of the second row will be equal to the least common multiple of the first row, each number of the second row will divide the corresponding number of the first row, and finally, the product of the numbers of the second row will be equal to the product of the numbers of the first row divided by $d$.

Now if $a_{h}$ and $a_{k}$ have a greatest common divisor $d$ which is not prime to $\frac{a_{h}}{d}$, but the two numbers $d$ and $\frac{a_{h}}{d}$ have a greatest common divisor $\delta>1$, then it is easy to see that the greatest common 'divisor of $a_{h}$ and $\frac{a_{k}}{\delta}$ will be equal to $\frac{d}{\delta}$. In fact, both $a_{h}$ and $\frac{a_{k}}{\delta}$ are divisible by $\frac{d}{\delta}$, giving the quotients $\frac{a_{h}}{d} \cdot \delta$ and $\frac{a_{k}}{d}$. I say these two quotients will be prime to one another, for $\frac{a_{h}}{d}$ is prime to $\frac{a_{k}}{d}$, on account of $d$ being the greatest common divisor of $\alpha_{h}$ and $a_{k}$, and $\delta$ being a divisor of $\frac{a_{h}}{d}$ will be prime to $\frac{a_{k}}{d}$ too, ${ }^{*}$ so that the product $\frac{a_{h}}{d} \times \delta$ is prime $\nmid$ to $\frac{a_{k}}{d}$. The least common multiple of $a_{h}$ and $\frac{a_{k}}{d}$ will be accordingly equal to $\frac{a_{h} \times \frac{a_{k}}{\delta}}{\frac{d}{\delta}}=\frac{a_{h} a_{k}}{d}$. So that if we replace the row of numbers

$$
a_{1}, a_{2}, \ldots a_{h} \ldots a_{k} \ldots a_{n}
$$

by the row of numbers

$$
a_{1}, a_{2}, \ldots a_{h} \ldots \frac{a_{k}}{\delta} \ldots a_{n},
$$

the least common multiple of the new row of numbers will be equal to the least common multiple of the first row, each

* Eucl. Elem. vIr. 23.

† Eucl. Elem. VII. 24. 
number of the second row will divide the corresponding number of the first row, and finally, the product of the numbers of the second row will be equal to the product of the numbers of the first row divided by $\delta$.

We come to the conclusion that so long as there are two numbers in the row

$$
a_{1}, a_{2}, \ldots a_{n}
$$

admitting a greatest common divisor larger than unity, the row can be replaced by another row

$$
a_{1}^{\prime}, a_{2}^{\prime}, \ldots a_{n}{ }^{\prime} \text {, }
$$

so that the least common multiple of the second row will be the same as the least common multiple of the first row, each number of the second row will divide the corresponding number of the first row, and, finally, the product of the numbers of the second row will be less than the product of the numbers of the first row. If we repeat the operation so long as the new row has numbers admitting a greatest common divisor larger than unity, we must finally come to a row of numbers

$$
b_{1}, b_{2}, \ldots b_{n}
$$

no two of which have a common divisor; otherwise the operation could be always continued, which is impossible on account of the diminishing of the product of the numbers at each successive operation. We shall have accordingly

$$
m=b_{1} b_{2} \ldots b_{n} \text {, }
$$

and the factors will have the required property.

It is now easy to prove the theorem, which by using the preceding notations can be expressed as follows:

$$
|(a, b),(a, c),(a, d),(a, e), \ldots|=(a,|b c d e \ldots|) .
$$

In fact, I put

$$
|b, c, d, e, \ldots|=b_{1} c_{1} d_{1} e_{1} \ldots
$$

so that $b_{1}, c_{1}, d_{1}, e_{1}, \ldots$ are prime each to each; and besides, $b_{1}$ divides $b, c_{1}$ divides $c, d_{1}$ divides $d, e_{1}$ divides $e$, etc. Then

$$
\begin{aligned}
& (a,|b c d e \ldots .|)=\left(a, b_{1} c_{1} d_{1} e_{1} \ldots\right)= \\
& =\left(a, b_{1}\right) \times\left(a, c_{1}\right) \times\left(a, d_{1}\right) \times\left(a, e_{1}\right) \ldots .
\end{aligned}
$$

Now as each of the numbers

divides

$$
(a, b),(a, c),(a, d),(a, e), \ldots
$$

$$
(a,|b c d e \ldots|) \text {, }
$$


their least common multiple will divide it too, so that the left side of the equality which it is required to prove divides the right side. But if we write down the two rows

$$
\begin{gathered}
\left(a, b_{1}\right), \quad\left(a, c_{1}\right), \quad\left(a, d_{1}\right), \quad\left(a, e_{1}\right) \ldots, \\
(a, b), \quad(a, c), \quad(a, d), \quad(a, e) \ldots,
\end{gathered}
$$

each number of the first row will divide the corresponding number of the second row, and accordingly the least common multiple of the numbers of the first row, that is to say, $(a,|b c d e \ldots|)$ will divide the least common multiple of the numbers of the second row, which we designated by

$$
\mid(a, b),(a, c),(a, d),(a, e) \ldots . .
$$

So that the right side of the equality which it is required to prove divides the left side. Accordingly the two sides are really equal.*

When we have more than two numbers, say $n$,

$$
a_{1}, a_{2}, \ldots a_{n},
$$

it is found convenient to consider besides the least common multiple and the greatest common divisor $n-2$ intermediate numbers. We might have arrived at such an extension of the original Euclidian theory by generalizing the geometrical interpretation given above, but I shall confine myself in this sketch to an arithmetical exposition of the subject. Let us put

$$
\begin{aligned}
& e_{n}=\left|a_{1}, a_{2}, \ldots a_{n}\right| \\
& e_{n-1}=\mid\left(a_{1}, a_{2}\right), \ldots\left(a_{1}, a_{n}\right),\left(a_{2}, a_{3}\right), \ldots\left(a_{2}, a_{n}\right), \ldots \\
& \left(a_{n-1}, a_{n}\right) \mid \\
& e_{n-}=\mid\left(a_{1}, a_{2}, a_{3}\right), \ldots\left(a_{1}, a_{4}, a_{n}\right),\left(a_{1}, a_{3}, a_{4}\right), \ldots \\
& \left(a_{1}, a_{3}, a_{12}\right),\left(a_{2}, a_{3}, a_{4}\right) \ldots\left(a_{2}, a_{3}, a_{n}\right) \ldots \\
& \left(a_{n-2}, a_{n-1}, a_{n}\right) \\
& e_{1} \dot{r}=\left|\left(\dot{a_{1}}, a_{2}, \ldots \cdot a_{n}\right)\right|=\left(a_{1}, \dot{a_{2}}, \ldots . \dot{a_{n}}\right) \text {. }
\end{aligned}
$$

Then it is easy to see that each $e_{k}$ is a multiple of $e_{k-1}$ So, for instance, $e_{n}$ is a multiple of $e_{n-1}$, because

$$
\begin{aligned}
& \left(a_{1}, a_{2}\right),\left(a_{1}, a_{s}\right), \ldots\left(a_{1}, a_{n}\right) \text { divide } a_{1} \\
& \left(r, a_{3}\right),\left(a_{2}, a_{4}\right), \ldots\left(a_{2}, a_{n}\right) \text { divide } a_{2} \\
& \cdot . \quad \cdot \quad \cdot . \\
& \left(a_{n-1}, a_{n}\right) \text { divides } a_{n-1} .
\end{aligned}
$$

* Grassmann's Arithmetik, No. 103. 
By using the rule that while forming the least common multiple of a lot of numbers any numbers of the lot may be replaced by their least common multiple, we arrive at the following expressions for our numbers $e_{n}, e_{n-1}, \ldots e_{1}$ :

$$
\begin{aligned}
& e_{n}=\left|a_{1}, a_{2}, \ldots a_{n}\right| \\
& e_{n-1}=\mid\left(a_{1},\left|a_{2}, \ldots a_{n}\right|\right),\left(a_{2},\left|a_{3} \ldots a_{n}\right|\right), \ldots \\
& \left(a_{n-1}, a_{n}\right) \\
& e_{n-2}=\mid\left(a_{1}, a_{2},\left|a_{3}, \ldots a_{n}\right|\right),\left(a_{1}, a_{3},\left|a_{4}, \ldots a_{n}\right|\right), \ldots \\
& \left(a_{1}, a_{n-1}, a_{n}\right), \quad\left(a_{2}, a_{8},\left|a_{4}, \ldots a_{n}\right|\right) \text {, } \\
& \left(a_{2}, a_{4},\left|a_{6}, \ldots a_{n}\right|\right), \ldots\left(a_{2}, a_{n-1}, a_{n}\right), \ldots \\
& \left(a_{n-2}, a_{n-1}, a_{n}\right)
\end{aligned}
$$

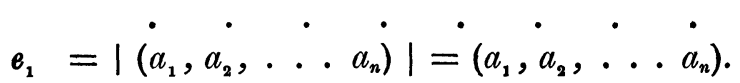

It follows that while $e_{n}$ is the least common multiple of $n$ numbers

$$
a_{1}, a_{2}, \ldots a_{n},
$$

$e_{n-1}$ is the least common multiple of $n-1$ numbers.

$$
\begin{gathered}
b_{1}=\left(a_{1},\left|a_{2}, \ldots a_{n}\right|\right), b_{2}=\left(a_{2},\left|a_{3}, \ldots a_{n}\right|\right), \ldots \\
\quad b_{n-1}=\left(a_{n-1}, a_{n}\right) .
\end{gathered}
$$

Now I say that $e_{n-2}$ may be deduced from $e_{n-1}$ in the same way as $e_{n-1}$ is deduced from $e_{n}$; that is to say, that while

$$
\begin{aligned}
& e_{n-1}=\mid\left(a_{1},\left|a_{2}, \ldots . a_{n}\right|\right),\left(a_{2},\left|a_{3}, \ldots a_{n}\right|\right), \ldots \\
& \quad\left(a_{n-1}, a_{n}\right), \mid \\
& \text { we have also }
\end{aligned}
$$

$$
\begin{gathered}
e_{n-2}=\mid\left(b_{1},\left|b_{2}, \ldots b_{n-1}\right|\right),\left(b_{2},\left|b_{3}, \ldots b_{n-1}\right|\right), \ldots \\
\left(b_{n-2}, b_{n-1}\right) \mid .
\end{gathered}
$$

I shall put for the sake of abbreviation

$$
\begin{gathered}
h_{1}=\left(b_{1},\left|b_{2}, \ldots b_{n-1}\right|\right), h_{2}=\left(b_{2},\left|b_{3}, \ldots b_{n-1}\right|\right), \ldots \\
\quad h_{n-2}=\left(b_{n-1}, b_{n}\right) .
\end{gathered}
$$

Now it is easy to prove that $h_{1}$ is the least common multiple of the numbers

$$
\begin{aligned}
& \left(a_{1}, a_{2},\left|a_{3}, \ldots a_{n}\right|\right),\left(a_{1}, a_{3},\left|a_{4}, \ldots a_{n}\right|\right), \ldots \\
& \left(a_{1}, a_{n-1}, a_{n}\right) \text {. }
\end{aligned}
$$

In fact,

$h_{1}=\left(b_{1},\left|b_{2}, \ldots b_{n-1}\right|\right)=\left|\left(b_{1}, b_{2}\right),\left(b_{1}, b_{3}\right), \ldots\left(b_{1}, b_{n-1}\right)\right|$. 
And as

$$
\begin{aligned}
& \left(b_{1}, b_{2}\right)=\left(a_{1}, a_{2},\left|a_{3}, \ldots a_{n}\right|,\left|a_{2}, \ldots a_{n}\right|\right) \\
& =\left(a_{1}, a_{2},\left|a_{8}^{\prime}, \ldots a_{n}\right|\right) \\
& \left(b_{1}, b_{3}\right)=\left(a_{1}, a_{3},\left|a_{4}, \ldots a_{n}\right|\right) \\
& \left(b_{1}, \dot{b_{n-1}}\right)=\left(a_{1}, a_{n-1}, a_{n}\right) \text {, }
\end{aligned}
$$

our assertion is proved. In a similar way $h_{2}$ is the least common multiple of the numbers

$\left(a_{2}, a_{3}\left|a_{4} \ldots a_{n}\right|\right),\left(a_{2}, a_{4},\left|a_{6} \ldots a_{n}\right|\right), \ldots\left(a_{2}, a_{n-1}, a_{n}\right)$; and finally

$$
h_{n-2}=\left|\left(a_{n-2}, a_{n-1}, a_{n}\right)\right| \text {. }
$$

The two expressions for $e_{n-2}$ are accordingly equal. In the same way by putting

$c_{1}=\left(b_{1},\left|b_{2}, \ldots b_{n-1}\right|\right), c_{2}=\left(b_{2},\left|b_{3}, \ldots b_{n-1}\right|\right), \ldots$

we have

$$
c_{n-2}=\left(b_{n-2}, b_{n-1}\right) \text {, }
$$

$$
\begin{aligned}
& e_{n-2}=\left|c_{1}, c_{2}, \ldots c_{n-2}\right| \\
& \text { and } \\
& e_{n-3}=\mid \underset{\left(c_{n-3}, c_{n-2}\right) \mid}{\left(c_{1},\left|c_{2}, \ldots c_{n-2}\right|\right),\left(c_{2},\left|c_{3}, \ldots c_{n-2}\right|\right), \ldots,}
\end{aligned}
$$

So that each of the numbers $e_{n}, e_{n-1}, \ldots e_{1}$ is deduced from the preceding in a similar way, the number of the numbers on which we have to operate gradually diminishing by one from $n$ in the case of $e_{n}$ to unity in the case of $e_{1}$. The operation is an unsymmetrical one, but as it gives the same result as the symmetrical one, the lack of symmetry has no influence on the result

Let us take the following numbers, for instance:

$a_{1}=480, a_{2}=720, a_{3}=2700, a_{4}=3240, a_{5}=6750, a_{6}=11250$.

Then by using Euclid's rule for finding the greatest common divisor, the whole calculation can be performed in the following way:

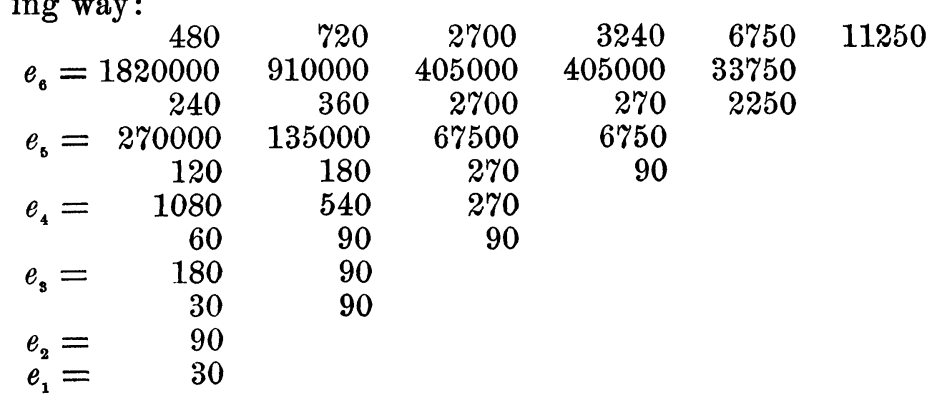


232 CONSTANCY OF FACTOR-GROUPS : HÖLDER'S THEOREM.[June,

The last number in the third row is the greatest common divisor of the two last numbers in the first row, and the last number in the second row is their least common multiple. The last number but one in the third row is the greatest common divisor of 3240 and 33750 , and 405000 is their least common multiple. The last number but two in the third row is the greatest common divisor of $2 \% 00$ in the first row and 405000 in the second row, and 405000 a little to the left is the least common multiple of the same numbers. The second number in the third row is the greatest common divisor of 720 and 405000 , and 910000 is their least common multiple. The first number in the third row is the greatest common divisor of 480 and 910000 , and $1820000=e_{6}$ is their least common multiple, etc., etc.

M. Stieltjes gives other expressions for the same numbers, for which I refer to his paper. We have now worked our way through the first chapter* with the figure of the great Greek mathematician overshadowing us all the while. The second chapter begins under the auspices of Gauss.

Clark University, May 23, 1895.

Joseph De Perott.

\section{NOTE ON HÖLDER'S THEOREM CONCERNING THE CONSTANCY OF FACTOR-GROUPS. $†$}

BX MR. GEORGE L. BROWN.

HöLDER's proof of the constancy of the factor-groups for the different series of composition of a group is based upon the following lemma:t If a group $G$ possesses two different maximal self-conjugate subgroups $A$ and $B$, having $C$ as theiv greatest common subgroup, then the quotient-groups $G \mid A$ and $B \mid C$, likewise $G \mid B$ and $A \mid C$, are holoedrically isomorphic.

The proof of this lemma may be very much simplified by making use of the following theorem, due to Giudice:\& If $A$ and $B$ are two commutative groups of orders $p$ and $q$ respertively, having $C$, of order $r$, as their greatest common subgroup, then the order of the group $F^{\prime}=\{A, B\}$ formed by combining the operations of $A$ and $B$ in every possible way is $\frac{p q}{r}$.

\footnotetext{
* I had no desire to exhaust the subject. The reader will find many propositions which I omitted in Grassmann's Arithmetik and Stieltjes' paper.

† Math. Annalen, vol. 34, pp. 34-37.

$\neq$ Hölder, 1. c., §9.

$\S$ Netto, Theory of Substitutions, $\S 38 ;$ F. Giudice, Palermo Rend. vol. 1 , pp. 222,223 .
} 